\title{
Comparative Hepatic and Intestinal Metabolism and Pharmacodynamics of Statins ${ }^{[\Phi}$
}

\author{
Anne M. Filppula, Päivi Hirvensalo, Heli Parviainen, Vilma E. Ivaska, K. Ivar Lönnberg, \\ Feng Deng, Jenni Viinamäki, Mika Kurkela, Mikko Neuvonen, and Mikko Niemi \\ Department of Clinical Pharmacology and Individualized Drug Therapy Research Program, Faculty of Medicine, \\ University of Helsinki, Helsinki, Finland (A.M.F., P.H., H.P., V.E.I., K.I.L., F.D., J.V., M.K., M.Ne., M.Ni.) and Department of Clinical \\ Pharmacology, HUS Diagnostic Center, Helsinki University Hospital, Helsinki, Finland (M.Ni.)
}

Received Feb 4, 2021; Accepted May 5, 2021

\section{ABSTRACT}

This study aimed to comprehensively investigate the in vitro metabolism of statins. The metabolism of clinically relevant concentrations of atorvastatin, fluvastatin, pitavastatin, pravastatin, rosuvastatin, simvastatin, and their metabolites were investigated using human liver microsomes (HLMs), human intestine microsomes (HIMs), liver cytosol, and recombinant cytochrome P450 enzymes. We also determined the inhibitory effects of statin acids on their pharmacological target, 3hydroxy-3-methylglutaryl-coenzyme A (HMG-CoA) reductase. In HLMs, statin lactones were metabolized to a much higher extent than their acid forms. Atorvastatin lactone and simvastatin (lactone) showed extensive metabolism [intrinsic clearance $\left(\mathrm{CL}_{\text {int }}\right)$ values of 3700 and $7400 \mu / \mathrm{min}$ per milligram], whereas the metabolism of the lactones of 2-hydroxyatorvastatin, 4-hydroxyatorvastatin, and pitavas-

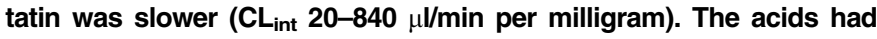
$\mathrm{CL}_{\text {int }}$ values in the range $<0.1-80 \mu / \mathrm{min}$ per milligram. In HIMs, only atorvastatin lactone and simvastatin (lactone) exhibited notable metabolism, with $\mathrm{CL}_{\text {int }}$ values corresponding to $20 \%$ of those observed in HLMs. CYP3A4/5 and CYP2C9 were the main statinmetabolizing enzymes. The majority of the acids inhibited HMG-CoA reductase, with $50 \%$ inhibitory concentrations of 4-20 nM. The present comparison of the metabolism and pharmacodynamics of the various statins using identical methods provides a strong basis for further application, e.g., comparative systems pharmacology modeling.

\section{SIGNIFICANCE STATEMENT}

The present comparison of the in vitro metabolic and pharmacodynamic properties of atorvastatin, fluvastatin, pitavastatin, pravastatin, rosuvastatin, and simvastatin and their metabolites using unified methodology provides a strong basis for further application. Together with in vitro drug transporter and clinical data, the present findings are applicable for use in comparative systems pharmacology modeling to predict the pharmacokinetics and pharmacological effects of statins at different dosages.

\section{Introduction}

3-Hydroxy-3-methylglutaryl-coenzyme A (HMG-CoA) reductase inhibitors (statins) are widely used in the treatment of hypercholesterolemia. Although statin drugs are effective and safe in most patients, many users experience poor efficacy or adverse drug reactions (Pazzucconi et al., 1995; Yebyo et al., 2019). The muscle toxicity of statins is a dose- and concentration-dependent phenomenon (Bradford et al., 1991; Dujovne et al., 1991), and the risk of toxicity increases along with the plasma concentrations of statins. This may be caused by drug-drug

This work was supported by the European Research Council Consolidator Grant [Grant Agreement 725249].

The authors declare no conflict of interest.

Parts of this work are included in the Master's Thesis of Heli Parviainen [Parviainen $\mathrm{H}$ (2020) Metabolism of Statins In Vitro. M.Sc. thesis, University of Helsinki, Helsinki, Finland] and Advanced Studies Thesis of Vilma E. Ivaska.

https://dx.doi.org/10.1124/dmd.121.000406.

S This article has supplemental material available at dmd.aspetjournals.org. interactions or inherited defects in proteins affecting statin disposition, such as CYP2C9 and 3A4, organic anion transporting polypeptide (OATP) 1B1, or breast cancer resistance protein (Thompson et al., 2003; Graham et al., 2004; Neuvonen et al., 2006; Pasanen et al., 2006; Keskitalo et al., 2009b; Hirvensalo et al., 2019).

Both acid and lactone forms of statins can interact with proteins involved in drug disposition. Lovastatin and simvastatin are administered as lactone prodrugs, whereas other statins are given in the active acid form. In the body, however, significant amounts of many statins are converted to their corresponding acid/lactone form (Neuvonen et al., 2006). Cytochrome P450 enzymes metabolize the lipophilic statin lactones more rapidly than statin acids (Fujino et al., 2004). Indirectly, uridine diphosphate-glucuronosyltransferase (UGT) enzymes may participate in lactone formation by mediating glucuronidation of statin acids. The acyl glucuronides formed in this process may then undergo spontaneous cyclization to the statin lactone (Prueksaritanont et al., 2002). CYP3A4 plays a key role in the elimination of atorvastatin, lovastatin, and simvastatin (Neuvonen et al., 2006). In turn, the $3 R, 5 S$ - and $3 S, 5 R$-enantiomers of fluvastatin are extensively biotransformed by

ABBREVIATIONS: $\mathrm{CL}_{\text {int }}$, intrinsic clearance; $\mathrm{C}_{\text {max }, \mathrm{u}}$, unbound peak concentration; $\mathrm{f}_{\mathrm{u} \text {,mic }}$, unbound fraction in microsomes; $\mathrm{HIM}$, human intestine microsome; HLC, human liver cytosol; HLM, human liver microsome; HMG-CoA, 3-hydroxy-3-methylglutaryl-coenzyme A; LC-MS/MS, liquid chromatography-tandem mass spectrometry; OATP, organic anion transporting polypeptide; PAPS, 3'-phosphoadenosine 5'-phosphosulfate; PBPK, physiologically based pharmacokinetic; PMSF, phenylmethylsulfonyl fluoride; SULT, sulfotransferase; UDPGA, uridine $5^{\prime}$-diphospho-glucuronic acid; UGT, uridine diphosphate-glucuronosyltransferase. 
CYP2C9 (Fischer et al., 1999; Hirvensalo et al., 2019). Pitavastatin, pravastatin, and rosuvastatin are excreted mainly unchanged (Neuvonen et al., 2006). Hence, the available statins differ significantly in their pharmacokinetic characteristics and susceptibility to altered metabolizing enzyme function.

Interestingly, the pharmacological target of statins, $\mathrm{HMG}-\mathrm{CoA}$ reductase, is expressed in the same location as the cytochrome P450 enzymes, the endoplasmic reticulum of hepatocytes (Corsini et al., 1995). Thus, this enzyme is also present in human liver microsomes (HLMs). Similar to cytochromes P450, the active site of HMG-CoA reductase faces the cytoplasm, and the enzyme uses NADPH for its catalytic activity (Corsini et al., 1995). Statin acids, which are structurally similar to its substrate HMG-CoA, competitively inhibit it in the nanomolar range, leading to a reduction of the HMG-CoA-mevalonate pathway. Also, several statin metabolites have been depicted to be inhibitors of this reaction, but in vitro data for, e.g., the 2- and 4-hydroxy metabolites of atorvastatin do not seem to be publicly available.

Many published physiologically based pharmacokinetic (PBPK) models of statins can be found in the literature, primarily aiming to evaluate their drug-drug interaction and pharmacogenetic properties. During the past two decades, PBPK modeling has evolved as an important tool in drug development, regulatory review, and clinical study design (Sager et al., 2015). PBPK modeling also holds the potential to become a valuable tool to inform drug and dosing selection in clinical practice (Johnson and Rostami-Hodjegan, 2011; Jamei, 2016; Venkatakrishnan and Rostami-Hodjegan, 2019). For such purposes, it is crucial that the data used for model development of a set of drugs are comparable and high in quality. Although the metabolism of statins has been widely investigated in vitro, only a few substrate depletion studies comparing the microsomal metabolism of different statins have been published (Fujino et al., 2004; Gertz et al., 2010, 2011; Varma et al., 2014). When appropriately applied, the substrate depletion approach results in intrinsic clearance $\left(\mathrm{CL}_{\text {int }}\right)$ values, which include all microsomal metabolic pathways of the tested compound (Obach, 1999), and can be scaled to hepatic metabolic clearance for PBPK modeling. Hence, to obtain comparable estimates of the metabolism of the widely used statins atorvastatin, fluvastatin, pitavastatin, pravastatin, rosuvastatin, and simvastatin and their metabolites, we carried out an extensive in vitro study in subcellular hepatic and intestinal fractions as well as in recombinant cytochrome P450 enzymes using low, clinically relevant statin concentrations. Moreover, to simulate the pharmacological response of statins in PBPK models, unbound statin concentrations in hepatocytes can be linked to in vitro measurements of their pharmacodynamic potency. Therefore, we also compared the inhibitory effects of these statins on their pharmacological target HMG-CoA reductase.

\section{Materials and Methods}

Chemicals and Reagents. Atorvastatin (acid), atorvastatin lactone, 2hydroxyatorvastatin (acid), 2-hydroxyatorvastatin lactone, 4-hydroxyatorvastatin (acid), 4-hydroxyatorvastatin lactone, mevalonate lactone, pravastatin (acid), rosuvastatin (acid), and all internal standards (Supplemental Table 1) were purchased from Toronto Research Chemicals (Toronto, Canada). $3 R, 5 S$-fluvastatin (acid), 3S,5R-fluvastatin (acid), pitavastatin (acid), and pitavastatin lactone were obtained from Santa Cruz Biotechnology (Dallas, Texas), and simvastatin acid and simvastatin (lactone) from SynFine Research (Ontario, Canada). Adenosine 3-phosphate $5^{\prime}$-phosphosulfate triethylammonium salt (PAPS), alamethicin, NADPH, uridine 5'-diphospho-glucuronic acid (UDPGA), and HMG-CoA reductase assay kits were obtained from Sigma-Aldrich (St. Louis, MO). HLMs (XTreme 200, a pool of 200 mixed-sex donors), human liver cytosol (HLC; XTreme 200, a pool of 200 mixed-sex donors), and human intestine microsomes (HIMs), both normal (a pool of 15 mixed-sex donors) and free from phenylmethylsulfonyl fluoride (PMSF; optimal in carboxylesterase activity; a pool of six mixed-sex donors), were purchased from Sekisui XenoTech (Tokai, Japan). The following recombinant EasyCYP Bactosomes were obtained from Cypex Ltd (Dundee, UK): CYP1A2R, CYP2A6BR, CYP2B6BR, CYP2C8BR, CYP2C9BHR, CYP2C19BR, CYP2D6R, CYP2E1BR, CYP2J2LR, CYP3A4BR, and CYP3A5BLR. Other chemicals were from Merck (Darmstadt, Germany).

Metabolism in Human Liver Microsomal Incubations. The metabolic depletion of statins was first measured in HLMs. With the exception for buffer controls, all incubations contained substrate, microsomes $(0.2 \mathrm{mg} / \mathrm{ml})$ in sodium phosphate buffer $(0.1 \mathrm{M}, \mathrm{pH} 7.4)$ with $\mathrm{MgCl}_{2}(5 \mathrm{mM})$. For simvastatin, the protein concentration was reduced to $0.1 \mathrm{mg} / \mathrm{ml}$ after the initial experiment. The depletion of each parent statin and its corresponding acid/lactone was studied in four different conditions: 1$)$ addition of NADPH $(1 \mathrm{mM})$ to the reaction mixture to measure the cytochrome P450-mediated metabolism; 2) addition of NADPH $(1 \mathrm{mM})$ and UDPGA $(5 \mathrm{mM})$ to measure both cytochrome P450- and UGTmediated metabolism; 3) no addition of cofactors (negative control); and 4) no addition of cofactors or microsomes (buffer control). In condition 2, alamethicin $(11 \mu \mathrm{g} / \mathrm{ml})$ was also included in the incubations to allow pore formation. The metabolism of the 2- and 4-hydroxy metabolites of atorvastatin were studied using conditions 1,3 , and 4 . The initial incubation concentrations of each substrate are listed in Supplemental Table 2 and Table 1.

All incubations were carried out once in triplicate on 96-well plates. The mixtures of substrate and microsomes (or only substrate in buffer in condition 4) were first preincubated for 15 minutes at $37^{\circ} \mathrm{C}$ and $350 \mathrm{rpm}$. After preincubation, cofactors were added to initiate the reactions in conditions 1 and 2. Samples were taken at $0,5,10,20,40$, and 60 minutes. For atorvastatin lactone and simvastatin, the sampling times were shorter $(0.5,1,3,5$, 7, and 10 minutes). Reactions were stopped by diluting samples $1: 3$ with ice-cold acetonitrile containing internal standard (1:2 for rosuvastatin) (Supplemental Table 1) and further handled as described below in the section Sample Processing and Analysis of Metabolism Samples.

Metabolism in Human Intestine Microsomal Incubations. The metabolic depletion of parent statins and their corresponding acid/lactone was also measured in HIMs. With the exception of buffer controls, all incubations contained substrate, microsomes $(0.2 \mathrm{mg} / \mathrm{ml})$ in sodium phosphate buffer $(0.1 \mathrm{M}$, $\mathrm{pH}$ 7.4) with $\mathrm{MgCl}_{2}(5 \mathrm{mM})$. For each substrate (incubation concentrations listed in Supplemental Table 2 and Table 1), the depletion was studied in four different conditions: 1) addition of NADPH $(1 \mathrm{mM})$ and UDPGA $(5 \mathrm{mM})$ to measure both cytochrome P450- and UGT-mediated metabolism (alamethicin $11 \mu \mathrm{g} / \mathrm{ml}$ also included), 2) no addition of cofactors (negative control), and 3) no addition of cofactors nor microsomes (buffer control). Furthermore, to screen for the potential effects of intestinal carboxylesterases on the metabolism of statins, each statin was also incubated with 4) $0.2 \mathrm{mg} / \mathrm{ml}$ PMSF-free HIMs (optimal in esterase activity). No cofactors were added to PMSF-free HIM incubations.

All incubations were carried out once in triplicate, preincubated, and stopped in a similar manner as described for the HLM incubations above. In conditions $1-3$, samples were taken at $0,5,10,20$, and 45 minutes. In condition 4 , only two samples were taken: at 0 and 25 minutes.

Metabolism Screening in Human Cytosolic Incubations. To screen for statin metabolism mediated by cytosolic enzymes, each statin was incubated with $\mathrm{HLC}(0.2 \mathrm{mg} / \mathrm{ml})$ in sodium phosphate buffer $\left(0.1 \mathrm{M}, \mathrm{pH}\right.$ 7.4) with $\mathrm{MgCl}_{2}$ $(5 \mathrm{mM})$ at $37^{\circ} \mathrm{C}$ and $350 \mathrm{rpm}$, either 1) without cofactors or 2) with the sulfotransferase (SULT) cofactor PAPS $(100 \mu \mathrm{M})$. Two samples were taken: 0 and 25 minutes. These conditions were selected to match those of the HIM screening (test setting 4 above). All incubations were carried out once in triplicate, and reactions were stopped as above.

Metabolism Screening with Recombinant Cytochrome P450 Enzymes. The depletion of statins was also measured in recombinant cytochrome P450 incubations. In the screening, the substrates were incubated with each one of 11 cytochrome P450 isoforms (CYP1A2, CYP2A6, CYP2B6, CYP2C8, CYP2C9, CYP2C19, CYP2D6, CYP2E1, CYP2J2, CYP3A4, and CYP3A5) separately at a protein concentration of $0.2 \mathrm{mg} / \mathrm{ml}$. The substrate concentrations were the same ones as in the HLM depletion experiment (Supplemental Table 2; Table 1). Samples were collected at 0,30 , and 60 minutes. All incubations were carried out once in triplicate, and reactions were stopped as above.

Inhibition of Metabolism in Microsomal Incubations. To confirm the results from the recombinant cytochrome $\mathrm{P} 450$ screening, inhibition studies in HLMs and HIMs were carried out for selected statins (HLMs: atorvastatin and atorvastatin lactone, $3 R, 5 S$ - and $3 S, 5 R$-fluvastatin, pitavastatin lactone, 


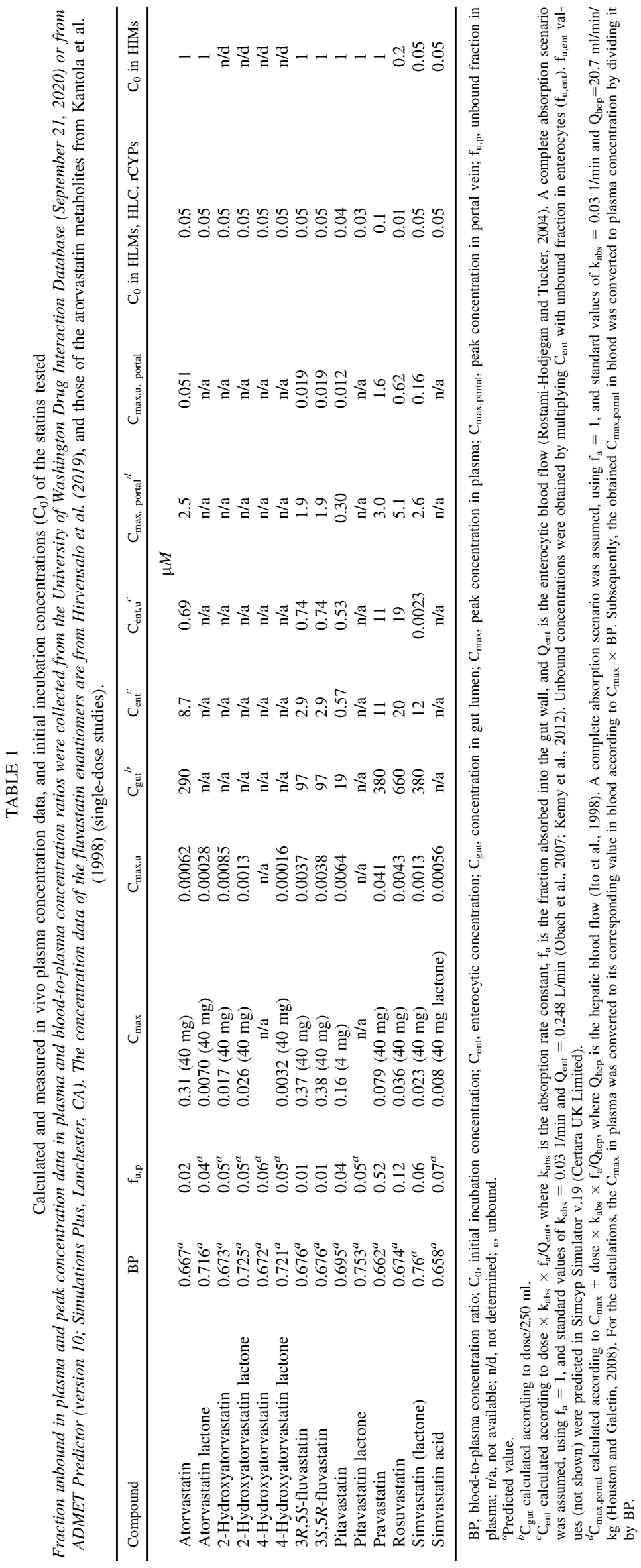


simvastatin, and simvastatin acid; HIMs: atorvastatin lactone and simvastatin). In HLMs, inhibition of CYP3A4 and cytochrome P450 isoforms causing $\geq 50 \%$ depletion at 60 minutes in the cytochrome $\mathrm{P} 450$ screening were tested. The substrate and NADPH concentrations used were identical to those used in the microsomal depletion experiments above (Supplemental Table 2; Table 1). The protein concentration was $0.2 \mathrm{mg} / \mathrm{ml}$ in atorvastatin and atorvastatin lactone experiments; $0.5 \mathrm{mg} / \mathrm{ml}$ in $3 R, 5 S$-fluvastatin and $3 S, 5 R$-fluvastatin, pitavastatin lactone, and simvastatin acid experiments; and $0.1 \mathrm{mg} / \mathrm{ml}$ in simvastatin experiments. Ketoconazole $(1 \mu \mathrm{M})$, montelukast $(5 \mu \mathrm{M})$, quinidine $(10 \mu \mathrm{M})$, and sulfaphenazole $(10$ $\mu \mathrm{M})$ were employed as competitive inhibitors of CYP3A4/5, CYP2C8, CYP2D6, and CYP2C9, respectively. Because of concerns regarding the selectivity of montelukast and quinidine, the effects of the time-dependent inhibitors gemfibrozil glucuronide (60 $\mu \mathrm{M}$; CYP2C8) and paroxetine (15 $\mu \mathrm{M}$; CYP2D6) were also tested. Whereas inhibitor and substrate were coincubated in direct inhibition experiments, the experiment with paroxetine included a 15-minute preincubation of inhibitor and NADPH and HLMs before addition of substrate. The experiment with gemfibrozil glucuronide was initiated by preincubating inhibitor with NADPH $(1 \mathrm{mM})$ in HLMs $(2 \mathrm{mg} / \mathrm{ml})$. After preincubation for 15 minutes, $10 \mu \mathrm{l}$ of the preincubation mix was moved to another well containing $190 \mu \mathrm{l}$ statin and NADPH $(1 \mathrm{mM})$ in buffer, diluting the protein concentration 20-fold. Samples in the inhibition experiments were taken at the same time points as in the HLM depletion experiment described above or at $0.5,2,4,6,8$, and 10 minutes (atorvastatin lactone). In HIM incubations, the protein concentration was $0.2 \mathrm{mg} / \mathrm{ml}$, and only the effects of ketoconazole $(1 \mu \mathrm{M})$ were tested. For atorvastatin lactone, samples were taken at $0,5,10,20$, and 45 minutes, and for simvastatin at $0,3.5,5,7.5$, and 10 minutes. All HLM and HIM incubations were carried out once in triplicate at $37^{\circ} \mathrm{C}$ and $350 \mathrm{rpm}$ and stopped as described above.

Determination of Unbound Fraction in Microsomes. Unbound fraction in microsomes $\left(\mathrm{f}_{\mathrm{u}, \mathrm{mic}}\right)$ values were measured using two-chambered rapid equilibrium dialysis devices (Thermo Scientific, Waltham, MA). HLMs $(0.2 \mathrm{mg} / \mathrm{ml} ; 0.1$ $\mathrm{mg} / \mathrm{ml}$ for simvastatin) or HIMs $(0.2 \mathrm{mg} / \mathrm{ml})$ in buffer $(200 \mu \mathrm{l})$ containing statin were transferred to one chamber, and $400 \mu \mathrm{l}$ buffer to the other, before incubation at $37^{\circ} \mathrm{C}$ for 4 hours on a shaker ( $300 \mathrm{rpm}$ ). At the end of the incubation, 25$\mu 1$ samples from the microsomal and buffer chambers were transferred to a 96well plate containing $100 \mu \mathrm{l}$ internal standard in acetonitrile. Blank buffer or blank HLMs or HIMs $(25 \mu \mathrm{l})$ were added to the samples from the microsomal or buffer chambers, respectively, to yield identical matrices. Samples were thereafter processed as described previously. The $\mathrm{f}_{\mathrm{u}, \mathrm{mic}}$ was calculated by dividing the statin concentration in buffer by that in the microsomal incubation mixture. To minimize potential metabolism by enzymes not dependent on external cofactors for their activity, old microsomes with several freeze and thaw cycles were used. In addition, they were allowed to incubate in room temperature 8 hours prior to the experiment. Denaturation was not carried out because of concerns that it would lead to conformational changes of the microsomal structure.

Inhibition of HMG-CoA. To investigate the inhibitory effects of statins on their pharmacological target HMG-CoA reductase, we first determined the time linearity of HMG-CoA metabolism at different enzyme concentrations (0.3-1.2 $\mu \mathrm{g} / \mathrm{ml})$. Herein, incubations containing HMG-CoA $(30 \mu \mathrm{M})$ and HMG-CoA reductase in phosphate buffer $(0.1 \mathrm{M})$ were first preincubated for 5 minutes at $37^{\circ} \mathrm{C}$ before addition of NADPH $(4.5 \mathrm{mM})$, which initiated the reactions. Reactions were stopped by placing $50-\mu \mathrm{l}$ samples in $150 \mu \mathrm{l}$ ice-cold acetonitrile containing mevalonolactone- $\mathrm{d} 3$ as the internal standard. Based on the obtained data, we determined the enzyme kinetics of HMG-CoA to its metabolite mevalonate by preincubating HMG-CoA $(5-240 \mu \mathrm{M})$ with HMG-CoA reductase $(0.9 \mu \mathrm{g} / \mathrm{ml})$ in phosphate buffer for 3 minutes. NADPH was added and the reactions were allowed to continue for 3 minutes before stopping them as described above. The same preincubation and incubation times $(3+3$ minutes) were also used in the final inhibition experiments. Herein, eight different concentrations of the statin acids $(0-500 \mathrm{nM})$ were simultaneously incubated with HMG-CoA at $20 \mu \mathrm{M}$ and HMG-CoA reductase $(0.9 \mu \mathrm{g} / \mathrm{ml})$ and NADPH in buffer. All incubations were performed once in triplicate. Use of recombinant enzyme was preferred over HLMs to avoid cytochrome P450-mediated metabolism of statins.

Sample Processing and Analysis of Metabolism Samples. After sample collection, metabolism samples were kept on ice for at least 10 minutes before centrifugation at $2000 \mathrm{~g}$ for 10 minutes. All samples were analyzed by liquid chromatography-tandem mass spectrometry (LC-MS/MS). The drug concentrations in HLM and recombinant cytochrome P450 enzyme incubations were determined using a Nexera X2 liquid chromatograph (Shimadzu, Kyoto, Japan) coupled to an API3000 tandem mass spectrometer (AB Sciex, Toronto, Ontario, Canada), as previously described for atorvastatin, fluvastatin, pitavastatin, pravastatin, rosuvastatin, and simvastatin (Keskitalo et al., 2009a,b,c; Itkonen et al., 2015; Lehtisalo et al., 2020). Part of the samples (HIM, HLC, rapid equilibrium dialysis, and some inhibition incubations) was analyzed using a Sciex 5500 Qtrap LC-MS/MS system (AB Sciex) interfaced with an ESI ion source. The chromatographic separation was carried out on a Luna Omega polar C18 column $(100 \times 2.1 \mathrm{~mm}$ I.D., $1.6-\mu \mathrm{m}$ particle size; Phenomenex, Torrance, CA) using 5 $\mathrm{mM}$ ammonium formate ( $\mathrm{pH} 3.9$, adjusted with $98 \%$ formic acid) as mobile phase $\mathrm{A}$ and acetonitrile as mobile phase $\mathrm{B}$. The flow rate and the column temperature were maintained at $300 \mu \mathrm{l} / \mathrm{min}$ and $40^{\circ} \mathrm{C}$. The following gradient conditions were applied: 1 minute at 20\% B on hold and then a linear ramp from $20 \%$ B to $40 \%$ B over 3 minutes followed by a second linear ramp to $90 \%$ B over 2 minutes and then 1 minute at $90 \%$ B before a re-equilibration step back to the initial conditions $(20 \% \mathrm{~B})$. The characteristic multiple reaction monitoring transitions for each analyte and internal standard are presented in Supplemental Table 1.

Sample Processing and Analysis of Pharmacodynamic Samples. After sample collection, samples were kept on ice, and $10 \mu \mathrm{HCl}(5 \mathrm{M})$ was added to ensure the lactonization of mevalonic acid into mevalonolactone (Honda et al., 2007). Samples were centrifuged at $2000 \mathrm{~g}$ for 10 minutes before LC-MS/MS analysis. Analytes were separated on Kinetex C18, $2.6 \mu \mathrm{m}, 100 \times 2.1 \mathrm{~mm}$ with SecurityGuard ULTRA C18 $2 \times 2.1 \mathrm{~mm}$ column (Phenomenex, Torrance, CA) by liquid chromatography (Nexera X2, ultra high performance liquid chromatography system; Shimadzu, Kyoto, Japan) with $0.05 \%$ formic acid as mobile phase A and acetonitrile as mobile phase $B$ at flow rate $0.2 \mathrm{ml} / \mathrm{min}$. The mobile phase B was kept at $30 \%$ for $0-1.5$ minutes, increased to $100 \%$ for $1.6-3.5$ minutes, and balanced at $30 \%$ before the next injection. The analytes were detected in a Shimadzu LCMS-8050 mass spectrometer (Shimadzu, Kyoto, Japan) operated in positive electrospray ionization mode (ESI+), and the multiple reaction monitoring transitions $[\mathrm{M}+\mathrm{H}]^{+}$were $\mathrm{m} / \mathrm{z}$ 130.9-43.0 for mevalonolactone and $\mathrm{m} / \mathrm{z}$ $133.9-45.95$ for the internal standard.

Data Analysis and In Vitro-In Vivo Extrapolation. The obtained data of the present in vitro experiments were analyzed using GraphPad Prism software (version 7.03; GraphPad Software, Inc., San Diego, CA). For depletion data, pseudo-first order depletion rate constants $\left(\mathrm{k}_{\mathrm{dep}}\right)$ were determined using nonlinear regression analysis $\left(\mathrm{C}=\mathrm{C}_{0} \times \mathrm{e}^{-\mathrm{kdep} \times \mathrm{t}}\right.$, where $\mathrm{C}$ is the observed concentration, $\mathrm{C}_{0}$ the initial concentration, and $\mathrm{t}$ is the incubation time). Only data points in the log-linear portion of each depletion curve were included in the analyses. Statin depletions observed in incubations with cofactors were corrected for depletions in incubations lacking cofactors (negative controls) by $\mathrm{k}_{\mathrm{dep}}$, corrected $=\mathrm{k}_{\mathrm{dep}}$, incubation -

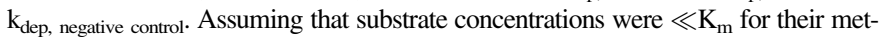
abolic pathways, their intrinsic clearance in depletion experiments was expressed as $\mathrm{CL}_{\text {int }}=\mathrm{k}_{\text {dep, corrected }} /[\mathrm{M}]$, where $[\mathrm{M}]$ is the microsomal protein concentration or cytochrome P450 concentration in recombinant enzyme incubations (Obach, 1999). Percent inhibition of statin depletion was calculated by comparing $C L_{\text {int }}$ values of incubations containing inhibitor to those lacking inhibitor. The mean of statin $\mathrm{CL}_{\text {int }}$ in the presence of each inhibitor was compared with the control using the Student's $t$ test in IBM SPSS Statistics (version 27.0; IBM Corp., Armonk, $\mathrm{NY}$ ). A $P$ value $<0.05$ was considered statistically significant. Unbound intrinsic clearance values, $\mathrm{CL}_{\mathrm{int}, \mathrm{u}}$, were calculated according to $\mathrm{CL}_{\mathrm{int}, \mathrm{u}}=\mathrm{CL}_{\mathrm{int}} / \mathrm{f}_{\mathrm{u}, \text { mic }}$. For pharmacodynamic data, statin $\mathrm{IC}_{50}$ values were determined using nonlinear regression in GraphPad Prism and related to their unbound peak concentrations in plasma $\left(\mathrm{C}_{\mathrm{max}, \mathrm{u}}\right)$ and in the portal vein.

\section{Results}

Metabolism in Microsomal and Cytosolic Incubations. In HLM incubations, statin lactones were metabolized to a much higher extent than their corresponding acid forms (Figs. 1A and 2A; Supplemental Table 3). In incubations with NADPH, atorvastatin lactone and simvastatin showed extensive metabolism $\left(\mathrm{CL}_{\mathrm{int}}\right.$ values of 3700 and 7400 $\mu \mathrm{l} /$ min per milligram), whereas the metabolism of the lactones of 2-hydroxyatorvastatin, 4-hydroxyatorvastatin, and pitavastatin was slower $\left(\mathrm{CL}_{\text {int }} 20-840 \mu \mathrm{l} / \mathrm{min}\right.$ per milligram). The statin acids had $\mathrm{CL}_{\text {int }}$ values in the range of $<0.1-80 \mu 1 /$ min per milligram. For most parent 
A

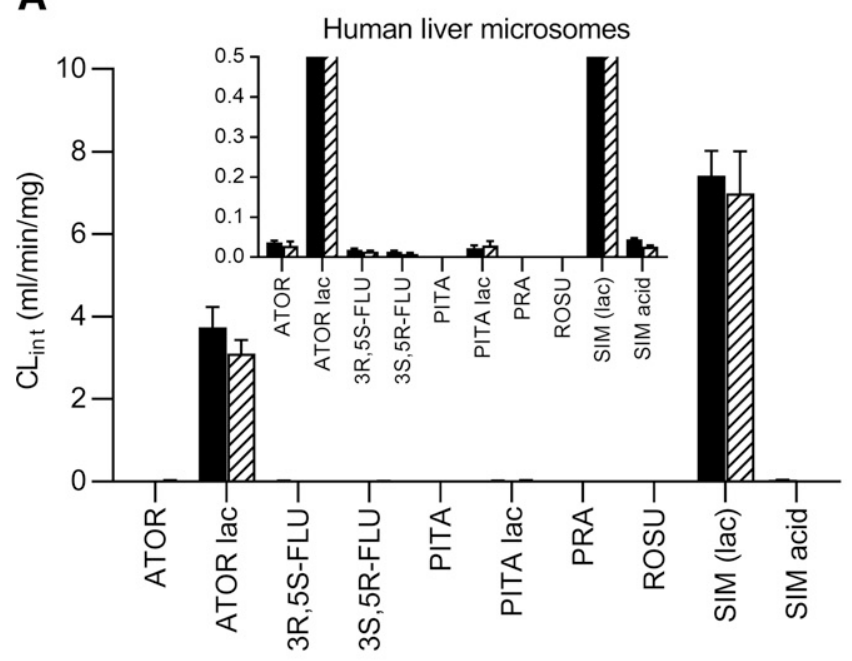

B

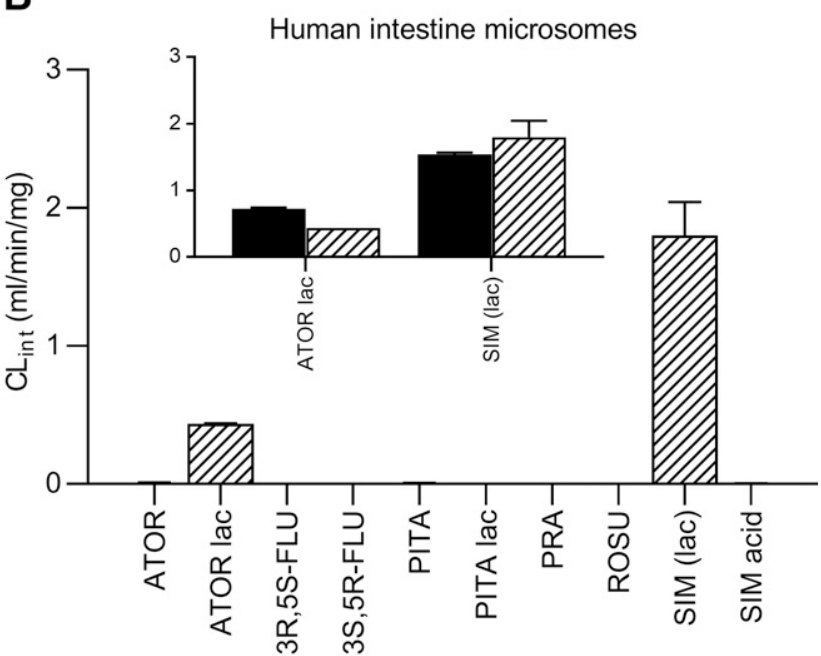

- NADPH

NADPH + UDPGA

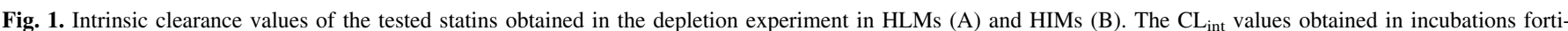

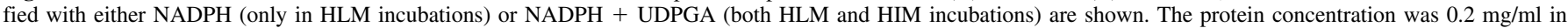

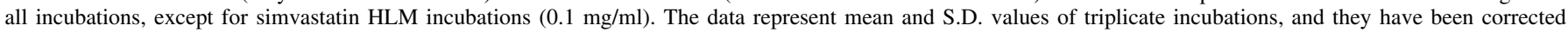

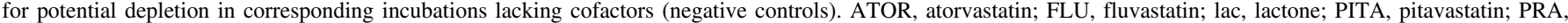
pravastatin; ROSU, rosuvastatin; SIM, simvastatin.

statins, the metabolism of both acid and lactone forms in NADPH + UDPGA incubations was of similar range or slightly slower than in NADPH incubations. No metabolism was observed in pitavastatin acid, pravastatin, and rosuvastatin incubations. In most HLM control incubations lacking cofactors (negative controls), the depletion of statins was $<20 \%$ at 60 minutes (Fig. 2A). However, in control incubations containing simvastatin and 4-hydroxyatorvastatin lactone, there seemed to be some cytochrome P450- and UGT-independent depletion occurring; for simvastatin, the depletion was $29 \%$ at 60 minutes, and for 4-hydroxyatorvastatin lactone, it was 32\%. Pitavastatin lactone exhibited large variation because of limited solubility, which was observed repeatedly across experiments. For most statins, the solubility seemed to be dependent on the presence of microsomal protein; the initial concentrations in buffer controls were often lower than in corresponding incubations containing microsomes, and the solubility seemed to increase with incubation time in buffer controls (data not shown). In the HLC screening, no clear statin metabolism was evident, either with or without PAPS (Fig. 2B).

In HIM incubations with NADPH and UDPGA, only atorvastatin lactone and simvastatin showed notable metabolism (Figs. 1B and 2C; Supplemental Table 4). The HIM $\mathrm{CL}_{\text {int }}$ values of atorvastatin lactone and simvastatin corresponded to approximately $20 \%$ of those obtained in HLM incubations. After incubation for 20-25 minutes, no clear metabolism was observed in HIM incubations lacking cofactors, except for a slight $17 \%$ decrease in simvastatin acid concentration in PMSF-free HIM incubations (Fig. 2C). For atorvastatin, atorvastatin lactone, and pravastatin, the decrease was $10 \%-12 \%$. For pitavastatin lactone, the concentration seemed to have increased with time. This is likely due to solubility issues, which were also observed in other incubations with pitavastatin lactone. $f_{u, m i c}$ values varied markedly between the statins (Supplemental Tables 3 and 4)

Metabolism Screening with Recombinant Cytochrome P450 Enzymes. In the recombinant cytochrome P450 screening, CYP3A4, CYP3A5, CYP2D6, CYP2C9, and CYP2C8 were the most active enzymes involved in statin metabolism (Fig. 2A). After incubation for 60 minutes, $\leq 50 \%$ of the initial substrate concentration remained in CYP3A4 incubations with atorvastatin lactone, 2-hydroxyatorvastatin lactone, 4-hydroxyatorvastatin lactone, pitavastatin lactone, simvastatin, and simvastatin acid. The same $(<50 \%$ remaining of the initial statin concentration) was observed in CYP3A5 incubations for atorvastatin lactone, 2-hydroxyatorvastatin lactone, and simvastatin; in CYP2D6 incubations for atorvastatin lactone, 2-hydroxyatorvastatin lactone, and pitavastatin lactone; in CYP2C9 incubations for the fluvastatin enantiomers; in CYP2C8 incubations for 2-hydroxyatorvastatin lactone and simvastatin; and in CYP1A2 and CYP2A6 incubations for 2-hydroxyatorvastatin lactone. Of note, whereas the lactones of atorvastatin, 2hydroxyatorvastatin and simvastatin, were completely metabolized by both CYP3A4 and CYP3A5, their acid forms were more prone to metabolism by CYP3A4 than by CYP3A5.

Inhibition of Metabolism in Microsomal Incubations. In HLMs, the CYP3A4 inhibitor ketoconazole completely $(>95 \%)$ inhibited the metabolism $\left(\mathrm{CL}_{\mathrm{int}}\right)$ of atorvastatin $(P<0.001)$ and atorvastatin lactone $(P=0.004)$ and that of simvastatin $(P=0.002)$ and simvastatin acid $(P<0.001)$ (Fig. 3). It had no effect $(<10 \%$ inhibition) on the depletion of $3 S, 5 R$-fluvastatin $(P=0.142)$, whereas it had a moderate effect on the $\mathrm{CL}_{\text {int }}$ of $3 R, 5 S$-fluvastatin (35\% inhibition, $P=0.027$ ) and that of pitavastatin lactone (18\%, $P=0.343)$. In turn, the CYP2C9 inhibitor sulfaphenazole inhibited the $\mathrm{CL}_{\text {int }}$ of $3 R, 5 S$-fluvastatin and $3 S, 5 R$-fluvastatin by $42 \%$ $(P<0.001)$ and $51 \%(P=0.010)$. The CYP2D6 inhibitor quinidine inhibited the depletion of atorvastatin lactone by $18 \%(P=0.093)$ and pitavastatin lactone by $18 \%(P=0.241)$. Paroxetine inhibited the depletion of atorvastatin lactone by $16 \%(P=0.106)$. The CYP2C 8 inhibitor montelukast inhibited the depletion of simvastatin by $25 \%(P=0.101)$, whereas gemfibrozil glucuronide had no effect on it $(P=0.840)$. In HIMs, ketoconazole inhibited the metabolism of atorvastatin lactone and simvastatin by $>95 \%(P<0.001$ and $P<0.001)$.

Interconversion between Acid and Lactone Forms in Microsomal and Cytosolic Incubations. Some interconversion between statin acid and lactone forms could be detected for atorvastatin, 
A

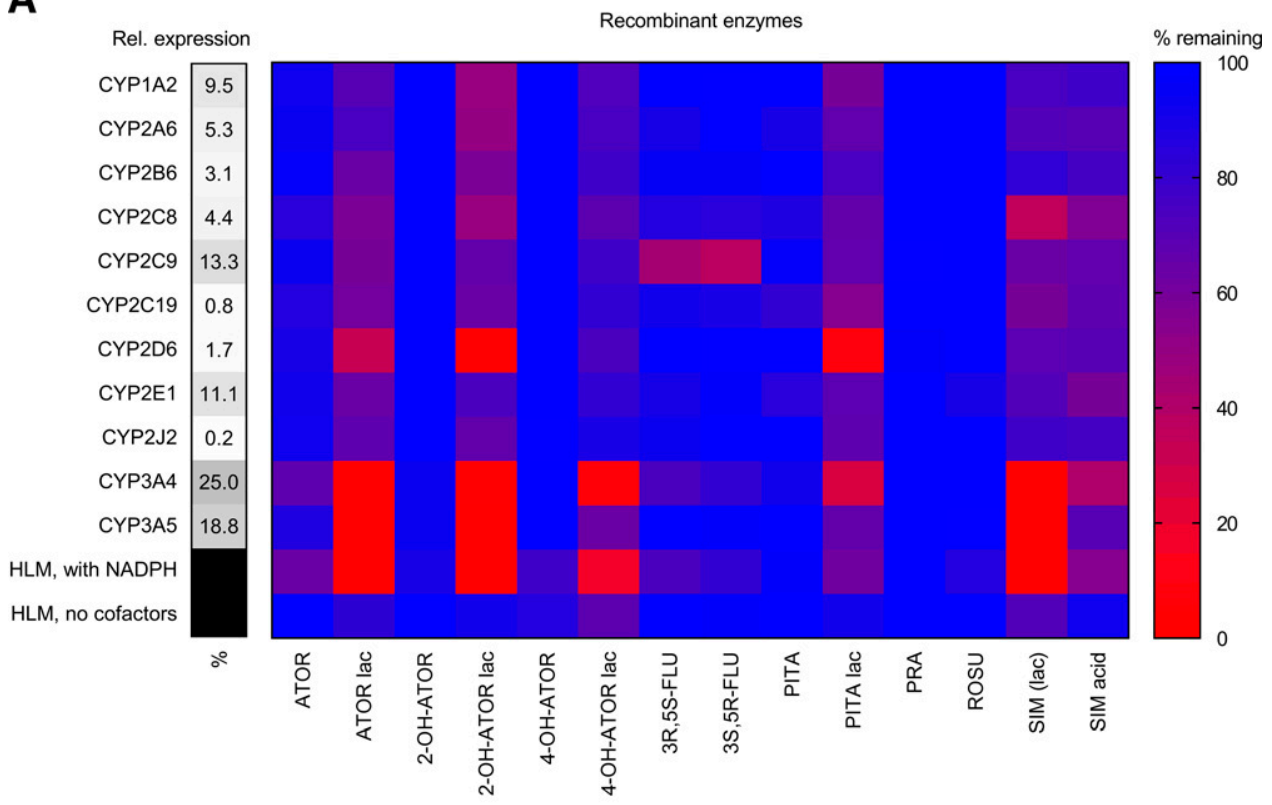

B

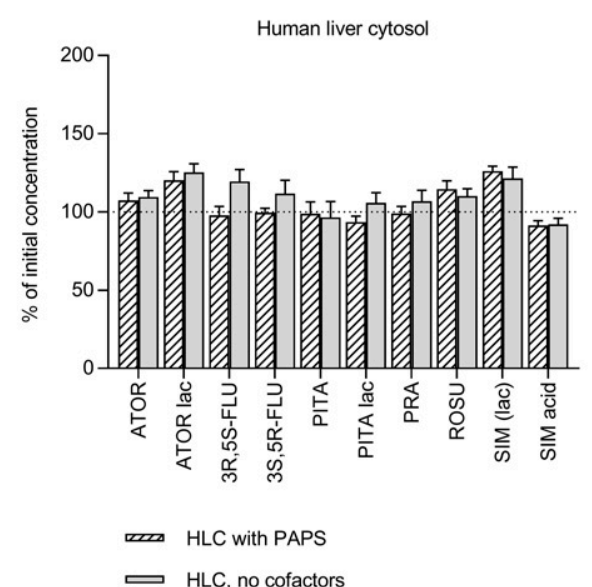

C

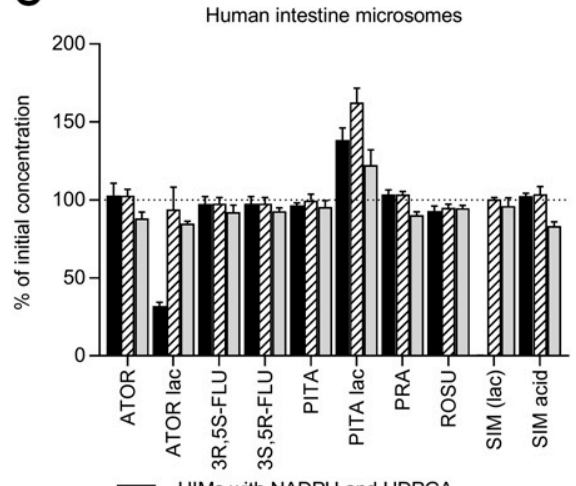

HIMs with NADPH and UDPGA

HIMs, no cofactors

PMSF-free HIMs, no cofactors

Fig. 2. Metabolism screening in recombinant cytochrome P450 (A), HLC (B), and HIM (C) incubations. In the recombinant cytochrome P450 screening, the mean statin concentrations remaining after a 60-minute incubation with 11 recombinant cytochrome P450 recombinant enzymes $(0.2 \mathrm{mg} / \mathrm{ml})$ and NADPH are shown. For comparison, the corresponding HLM data, with and without NADPH, are also included. The relative hepatic expression (\%) of each cytochrome P450 is shown in the panel to the left (calculated based on data available in Simcyp Simulator v.20; Certara UK Limited). In the HLC screening, no notable metabolism was observed after a 25-minute incubation of statins with HLC $(0.2 \mathrm{mg} / \mathrm{ml})$, with or without PAPS. In the HIM figure, the parent statin concentrations remaining after a 25 -minute incubation with PMSF-free HIMs $(0.2 \mathrm{mg} / \mathrm{ml})$ are shown (no cofactors included). In addition, 20-minute data from the depletion experiment in normal HIMs both with and without NADPH and UDPGA are also shown. The data presented represent mean and S.D. values of triplicate incubations. ATOR, atorvastatin; FLU, fluvastatin; lac, lactone; OH, hydroxy; PITA, pitavastatin; PRA, pravastatin; ROSU, rosuvastatin; SIM, simvastatin.

pitavastatin, and simvastatin. To examine the extent of interconversion in HIMs, we quantified the concentrations of the corresponding lactone in statin acid incubations and those of the corresponding acid in lactone incubations (Supplemental Fig. 1). For other incubations, the data described reflect qualitative data.

In HIMs supplemented with NADPH + UDPGA, lactone concentrations marginally increased in pitavastatin but not in atorvastatin incubations (Supplemental Fig. 1). In HLMs with NADPH and UDPGA, the lactone concentrations of both atorvastatin and pitavastatin slightly increased, but not in other incubations (NADPH, control). For simvastatin acid, there was no increase in lactone in HLMs and HIMs. In other incubations (PMSF-free HIMs, HLC \pm PAPS) with these statin acids, no increase in lactone was observed.

For statin lactones, there seemed to be a trend toward increase of acid concentrations in negative control (no cofactor) HLM and
HIM incubations, in PMSF-free HIMs (no cofactor added), and in HLC $( \pm$ PAPS $)$. More specifically, for atorvastatin lactone, acid concentrations increased up to $8 \%$ and $13 \%$ of the initial lactone concentration in NADPH + UDPGA and no cofactor HIM incubations, respectively (Supplemental Fig. 1). In NADPH incubations, no increase in acid concentrations was observed. In HLMs, acid concentrations increased in negative control incubations but not in those containing cofactors. In PMSF-free HIMs and HLC, the atorvastatin acid concentrations seemed to increase slightly.

For pitavastatin lactone, acid concentrations slightly increased in all incubations. For simvastatin, there seemed to be an increase in acid concentrations (up to $10 \%$ of the initial simvastatin concentration) in HIMs lacking cofactors but not in those containing cofactors (Supplemental Fig. 1). In HLMs, there was no increase in acid concentrations. In PMSF-free HIMs and HLC, the acid concentrations seemed to increase slightly. 
A

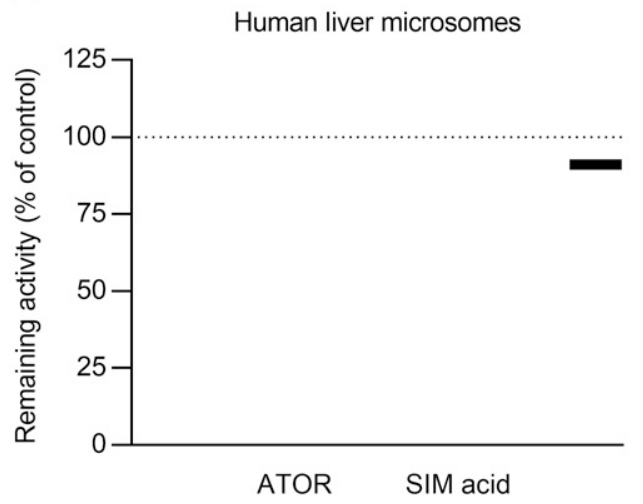

C

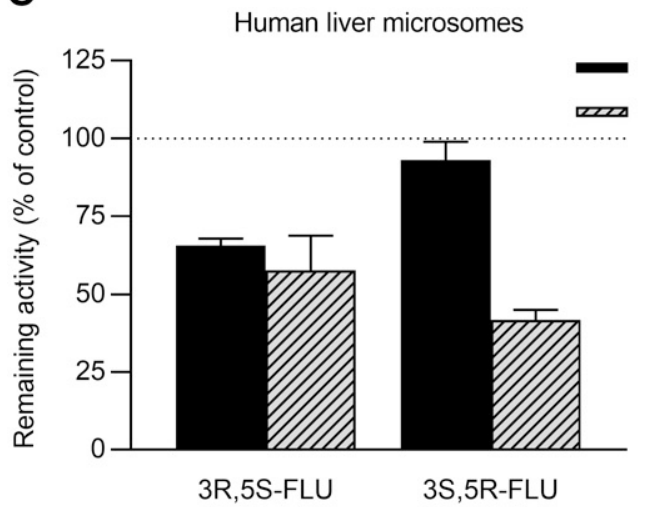

E

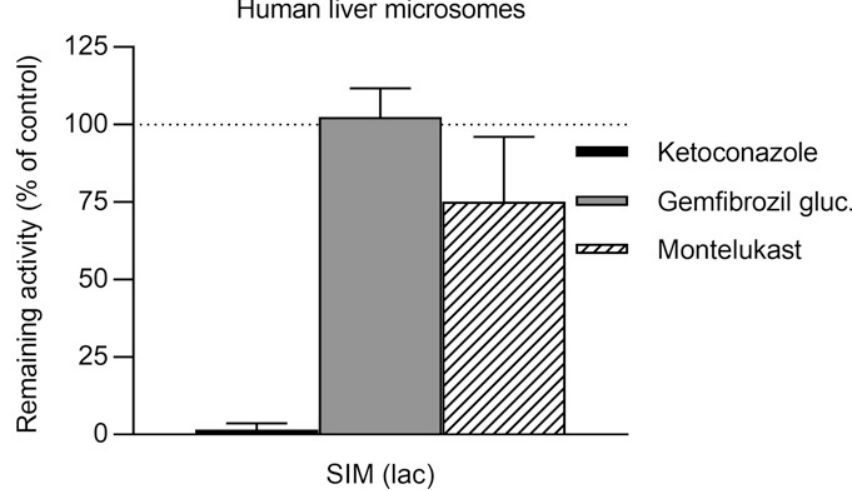

B

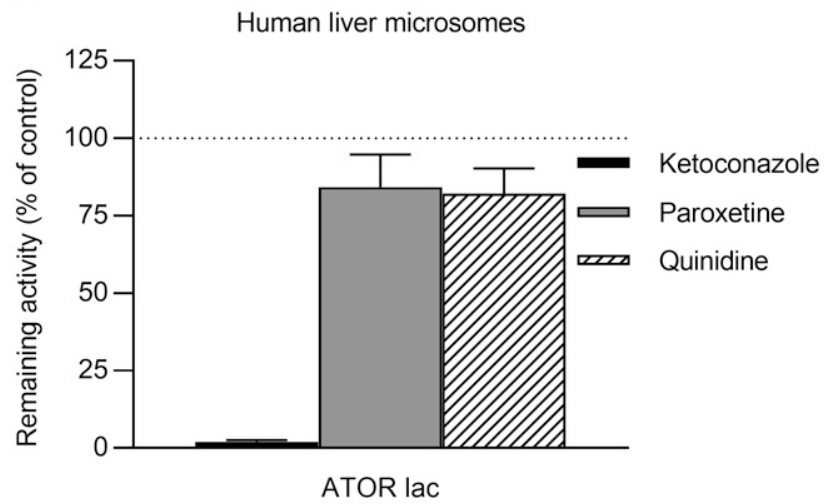

D

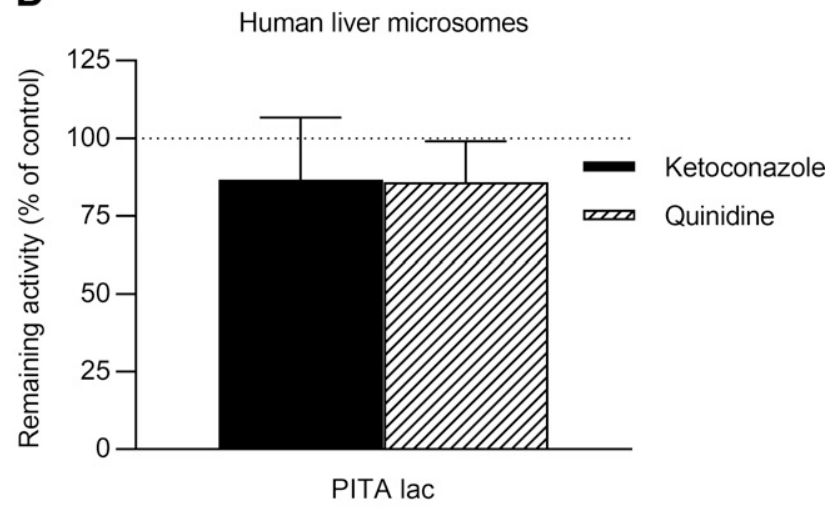

$\mathbf{F}$

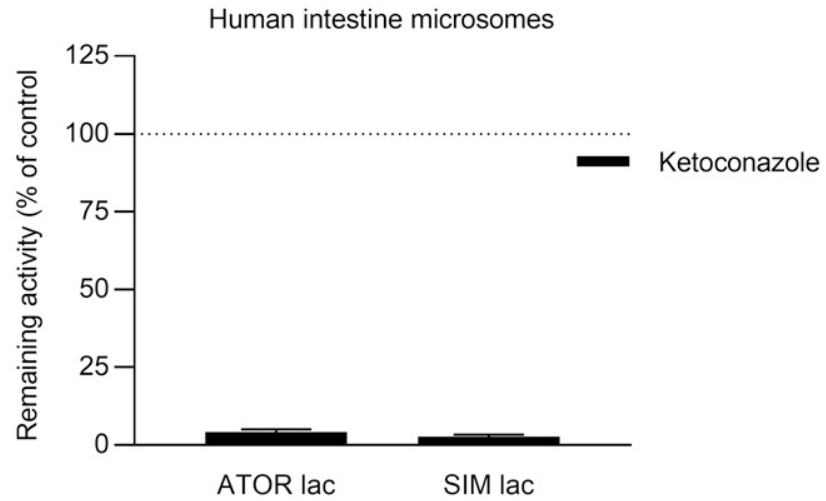

Fig. 3. Inhibition of statin metabolism by selected inhibitors in HLM and HIM incubations (A-F). The inhibitory effects of ketoconazole $1 \mu \mathrm{M}$ (CYP3A4/5), gemfibrozil glucuronide $60 \mu \mathrm{M}$ (CYP2C8), montelukast $5 \mu \mathrm{M}$ (CYP2C8), quinidine $10 \mu \mathrm{M}$ (CYP2D6), paroxetine $15 \mu \mathrm{M}$ (CYP2D6), and sulfaphenazole 10 $\mu \mathrm{M}$ (CYP2C9) on the depletion of statins were tested in HLMs $(0.5 \mathrm{mg} / \mathrm{ml}$ protein in fluvastatin, pitavastatin lactone, and simvastatin acid incubations; $0.2 \mathrm{mg} / \mathrm{ml}$ in atorvastatin acid and lactone incubations; $0.1 \mathrm{mg} / \mathrm{ml}$ in simvastatin incubations) and HIMs $(0.2 \mathrm{mg} / \mathrm{ml})$. The values indicate the remaining metabolic activity in the presence of inhibitor and are the mean and S.D. values of triplicate incubations. The values have been corrected for potential depletion in corresponding incubations lacking cofactors (negative controls). Pitavastatin lactone exhibited large variation due to limited solubility, which was observed repeatedly across experiments. Incubation with paroxetine was also carried out for pitavastatin lactone, but its depletion after the preincubation step was too low to be measured. ATOR, atorvastatin; FLU, fluvastatin; gluc, glucuronide; lac, lactone; PITA, pitavastatin; PRA, pravastatin; SIM, simvastatin.

Inhibition of HMG-CoA. In enzyme kinetic experiments, the kinetics of HMG-CoA was best described by a substrate inhibition model, with $\mathrm{K}_{\mathrm{m}}, \mathrm{K}_{\mathrm{i}}$, and $\mathrm{V}_{\max }$ values corresponding to $13 \mu \mathrm{M}, 105 \mu \mathrm{M}$, and $1,582 \mathrm{nmol} / \mathrm{min}$ per milligram (Supplemental Fig. 2). In pharmacodynamic experiments, the majority of the statin acids tested inhibited the HMG-CoA-mevalonate reaction with $\mathrm{IC}_{50}$ values in the range of 4-20 $\mathrm{nM}$ (Fig. 4; Table 2). The $\mathrm{IC}_{50}$ of $3 R, 5 S$-fluvastatin was $9 \mathrm{nM}$, whereas that of its $3 S, 5 R$ enantiomer approximated to $100 \mathrm{nM}$. Similarly, the $\mathrm{IC}_{50}$ of 2-hydroxyatorvastatin acid was $12 \mathrm{nM}$, but that of 4-hydroxyatorvastatin acid corresponded to $\sim 100 \mathrm{nM}$. Although the $\mathrm{IC}_{50}$ values of most statins were relatively close to their typical unbound peak concentrations in plasma (Fig. 4), those of 3S,5R-fluvastatin and simvastatin acid exceeded clinically relevant concentrations of $3 S, 5 R$-fluvastatin and simvastatin acid by 26 - to 35 -fold.

\section{Discussion}

This study aimed to comprehensively investigate and compare the metabolism and pharmacodynamics of 14 statins or statin metabolites 


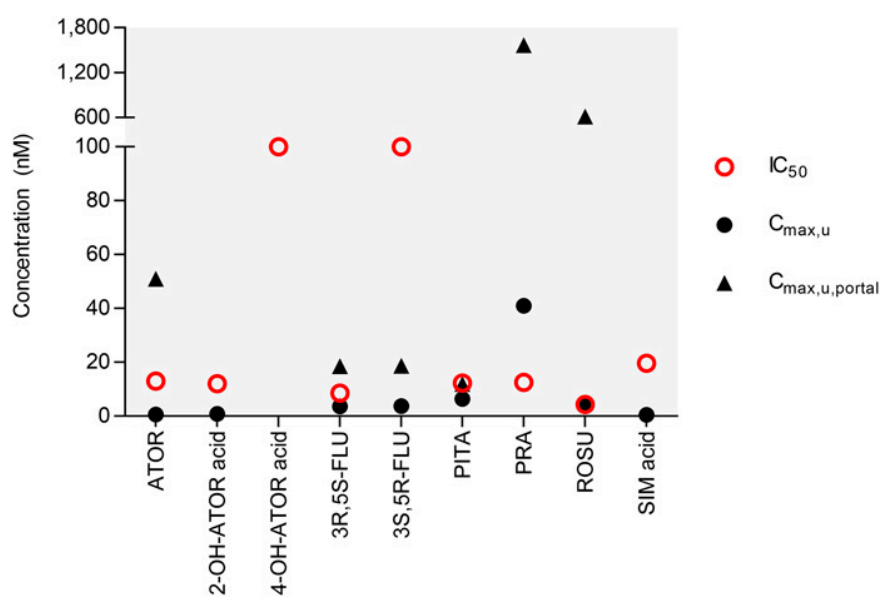

Fig. 4. Pharmacodynamic $\mathrm{IC}_{50}$ values of statin acids in comparison with their plasma concentrations. To test the inhibitory effects of the acid forms of the statins on their pharmacological target, eight different concentrations of the test compound were incubated with HMG-CoA $(20 \mu \mathrm{M})$ and HMG-CoA reductase $(0.9 \mu \mathrm{g} / \mathrm{ml})$ for 3 minutes. The results shown describe mean values of triplicate incubations (duplicate incubations for pitavastatin). The $\mathrm{C}_{\max , \mathrm{u}}$ values in plasma and in the portal vein $\left(\mathrm{C}_{\text {max,u,portal }}\right)$ are from Table 1 . As described therein, the $\mathrm{C}_{\text {max,u,portal }}$ concentrations were calculated assuming a complete absorption scenario. ATOR, atorvastatin; FLU, fluvastatin; OH, hydroxy; PITA, pitavastatin; PRA, pravastatin; ROSU, rosuvastatin; SIM, simvastatin.

using identical methods. Our collective data from HLM, HIM, HLC, and recombinant enzyme incubations underline the importance of cytochrome P450 enzymes in statin metabolism. According to our findings, CYP3A4/5 (simvastatin, atorvastatin) and CYP2C9 (fluvastatin) are the main statin-metabolizing enzymes in the liver. In HLMs, statin lactones were metabolized to a much higher extent than their corresponding acids. No or very little statin depletion occurred in the liver cytosol. In HIMs, CYP3A4/5 extensively metabolized atorvastatin lactone and simvastatin. No intestinal or hepatic metabolism was observed for pitavastatin, pravastatin, and rosuvastatin. In the pharmacodynamic experiment, the majority of the statin acids inhibited HMG-CoA reductase with $\mathrm{IC}_{50}$ values of 4-20 nM. Collectively, our findings are applicable for use in comparative systems pharmacology modeling of statins.

Although statin metabolism has been widely investigated in vitro, only a few depletion studies comparing the metabolism of statins have been published (Fujino et al., 2004; Gertz et al., 2010, 2011; Varma et al., 2014), and none with the present palette of statins. Our HLM and HIM $\mathrm{CL}_{\text {int }}$ values were generally within 2-fold of the previous $\mathrm{CL}_{\mathrm{int}}$

\section{TABLE 2}

The in vitro pharmacodynamic effects of statin acids To test the inhibitory effects of the acid forms of the statins on their pharmacological target, eight different concentrations of the test compound were incubated with $\mathrm{HMG}$-CoA $(20 \mu \mathrm{M})$ and $\mathrm{HMG}$-CoA reductase $(0.9 \mu \mathrm{g} / \mathrm{ml})$ for $3 \mathrm{~min}$. The data represent mean and S.D. values of triplicate incubations (duplicate incubations for pitavastatin).

\begin{tabular}{lc}
\hline Compound & $\mathrm{IC}_{50}$ \\
\hline & $n M$ \\
Atorvastatin & $13.1 \pm 3.2$ \\
2-Hydroxyatorvastatin & $12.1 \pm 4.2$ \\
4-Hydroxyatorvastatin & $\sim 100^{a}$ \\
$3 R, 5 S$-fluvastatin & $8.58 \pm 2.61$ \\
$3 S, 5 R$-fluvastatin & $\sim 100^{a}$ \\
Pitavastatin & $12.4 \pm 1.8$ \\
Pravastatin & $12.6 \pm 3.7$ \\
Rosuvastatin & $4.37 \pm 1.13$ \\
Simvastatin acid & $19.7 \pm 2.0$ \\
\hline
\end{tabular}

${ }^{a}$ The highest concentrations tested were 100 and $500 \mathrm{nM}$. values from depletion studies. In agreement with previous knowledge (Fujino et al., 2004), the lactones were metabolized more extensively than their acid forms. Atorvastatin lactone and simvastatin showed extensive metabolism, with HLM CL $\mathrm{int}_{\text {t }}$ values of 3700 and $7400 \mu \mathrm{l} / \mathrm{min}$ per milligram, whereas those of the lactones of 2-hydroxyatorvastatin, 4-hydroxyatorvastatin, and pitavastatin were in the range of $20-840 \mu \mathrm{l} /$ min per milligram. The statin acids had $\mathrm{CL}_{\text {int }}$ values below $80 \mu \mathrm{l} / \mathrm{min}$ per milligram, with pitavastatin acid, pravastatin, and rosuvastatin showing negligible metabolism. In HIMs, only atorvastatin lactone and simvastatin exhibited notable metabolism, with $\mathrm{CL}_{\text {int }}$ values corresponding to $20 \%$ of those obtained in HLMs. As compared with incubations with NADPH as the single cofactor, UDPGA addition did not result in an increased depletion. This indicates that the role of UGTs in the overall statin metabolism is relatively small.

To verify the cytochromes $\mathrm{P} 450$ involved in statin metabolism and to evaluate the potential role of the poorly characterized CYP2J2, we carried out an extensive screening in a panel of 11 recombinant cytochromes P450. Here, CYP3A4, CYP3A5, CYP2D6, CYP2C9, and CYP2C8 were the most active enzymes. CYP2J2 exhibited some activity toward atorvastatin lactone, 2-hydroxyatorvastatin lactone, pitavastatin lactone, simvastatin, and simvastatin acid. On the other hand, these compounds were metabolized to various degrees by almost all cytochromes P450 tested. Our inhibition data in HLMs and HIMs demonstrated a major role for CYP3A4/5 in the metabolism of atorvastatin, atorvastatin lactone, simvastatin acid, and simvastatin, in line with clinical observations (Neuvonen et al., 2006). For fluvastatin, CYP2C9 was of greater importance than CYP3A4. Furthermore, CYP2C9 was more prominent in the metabolism of $3 S, 5 R$-fluvastatin than in that of its enantiomer, as described earlier (Hirvensalo et al., 2019). Pitavastatin lactone was markedly metabolized by CYP2D6 and CYP3A4 in the screening, but its depletion was inhibited only moderately by the corresponding inhibitors in HLMs. Overall, our data demonstrating key roles for CYP3A4 and CYP2C9 in statin metabolism are line with previous literature (Fujino et al., 2004; Neuvonen et al., 2006).

The interconversion between the acid and lactone forms of statins introduces an additional level of complexity into statin pharmacokinetics. The process can be enzyme-mediated, spontaneous, or pH-driven and occur in both the blood stream and hepatocytes (Jemal et al., 1999; Billecke et al., 2000; Prueksaritanont et al., 2002; Hoffmann and Nowosielski, 2008; Li et al., 2019). In line with previous studies (Prueksaritanont et al., 2002; Fujino et al., 2003; Yamada et al., 2003), we observed slight increase in lactone concentrations in some statin acid incubations with UDPGA. The underlying mechanism is likely UGT-mediated glucuronidation, followed by spontaneous lactonization (Prueksaritanont et al., 2002). For statin lactones, there was a trend toward increase of acid concentrations in negative control microsomal incubations, suggesting a role for enzymes that do not require external cofactors for their activity, such as carboxylesterases (Liederer and Borchardt, 2006). In PMSF-free HIMs, however, less than $10 \%$ of the initial lactone concentrations had been depleted at 25 minutes. For comparison, in HIMs fortified with NADPH and UDPGA, only $32 \%$ and $<1 \%$ of the initial atorvastatin lactone and simvastatin (lactone) remained at 20 minutes. Thus, the role of intestinal membrane-bound esterases in lactone depletion seems to be negligible. There are contradictory findings on the role of human carboxylesterases in the conversion of simvastatin to its acid form; however, these enzymes seem to catalyze this process in blood (Vickers et al., 1990; Wang et al., 2015; Li et al., 2019). Together with SULTs, carboxylesterases are also present in smaller fractions in the cytosol (Boberg et al., 2017). In our HLC incubations ( \pm PAPS), no statin showed a $>10 \%$ reduction in their concentrations at 25 minutes. No depletion was observed for pravastatin, which has been reported to be a SULT substrate in rat liver (Kitazawa et al., 1993; Watanabe et al., 
2009). As a longer incubation time might have shown some involvement of cytosolic enzymes in statin metabolism, our HLC data should be interpreted with caution.

We used substrate depletion to obtain comparable estimates of the microsomal metabolism of each statin. The depletion approach operates under the assumption that the initial substrate concentration is well below $\mathrm{K}_{\mathrm{m}}\left(\mathrm{C}_{0} \ll \mathrm{K}_{\mathrm{m}}\right)$ (Obach, 1999). In our experiments, we attempted to use equal protein concentrations $(0.2 \mathrm{mg} / \mathrm{ml} ; 0.1 \mathrm{mg} / \mathrm{ml}$ for simvastatin) and low, clinically relevant statin concentrations. For most statins, an incubation concentration of $0.05 \mu \mathrm{M}$ was used. For pitavastatin acid and lactone and rosuvastatin the corresponding concentrations were slightly lower $(0.01-0.04 \mu \mathrm{M})$, and for pravastatin it was higher $(0.1$ $\mu \mathrm{M}$ ), based on initial estimations of typical unbound plasma concentrations. Regardless, in HLM incubations, the $\mathrm{C}_{0} \ll \mathrm{K}_{\mathrm{m}}$ criterion was fulfilled for all statins with $\mathrm{K}_{\mathrm{m}}$ values reported in the literature (Supplemental Table 2). Assuming a "worst case" scenario with complete absorption of the statin into enterocytes, our initial substrate concentrations in HIMs were in general 20-fold higher than those in HLMs. For the fluvastatin enantiomers, the concentrations used did not fulfill the $\mathrm{C}_{0} \ll \mathrm{K}_{\mathrm{m}}$ criterion in HIMs. However, no fluvastatin metabolism was observed in HIM incubations, and also the metabolism in HLMs was limited.

The present microsomal experiments included both buffer controls and negative controls. As most statins needed protein to dissolve in the incubations, it was not possible to distinguish between potential degradation of compound in buffer and depletion in incubations lacking cofactors. However, by correcting for depletion in negative control incubations, we were able to accurately measure the NADPH- and UDPGA-dependent microsomal metabolism. Use of microsomes instead of hepatocytes allowed us to measure metabolic $\mathrm{CL}_{\text {int }}$ values in a system stripped from confounding factors, such as drug transporters. Interestingly, although rosuvastatin is not metabolized in microsomes, there are data showing (very slow) rosuvastatin metabolism in hepatocytes (McCormick et al., 2000) . The statins are known substrates of drug transporters, in particular of OATP1B1 and breast cancer resistance protein (Pasanen et al., 2006; Keskitalo et al., 2009c; Giacomini et al., 2013). Consequently, the hepatic clearance of statins is a product of their metabolic $\mathrm{CL}_{\mathrm{int}}$ values in combination with their transporter $\mathrm{CL}_{\text {int }}$ values. Together with in vitro drug transport and pharmacodynamic data in combination with clinical data, our $\mathrm{CL}_{\mathrm{int}}$ values can be applied in systems pharmacology modeling of statin pharmacokinetics and effects.

In the present study, we also tested the inhibitory effects of the statin acids on their pharmacological target in the liver, HMG-CoA reductase. Although previous studies have determined these effects using radiometric assays or spectrophotometry to measure NADPH consumption (Kathawala, 1991; Holdgate et al., 2003; Perchellet et al., 2009), we used LC-MS/MS to measure mevalonolactone concentrations. Overall, our findings are in good agreement with literature data (Supplemental Table 5). Our $\mathrm{IC}_{50}$ value obtained for pravastatin $(13 \mathrm{nM})$, however, is 4- to 5-fold lower than previous measurements (McTaggart et al., 2001; Perchellet et al., 2009). The low value is supported by our preliminary experiment, in which the $\mathrm{IC}_{50}$ of pravastatin was $15 \mathrm{nM}$ (data not shown). 3R,5S-fluvastatin $\left(\mathrm{IC}_{50}=9 \mathrm{nM}\right)$ was about 12 times more active than its $3 S, 5 R$-enantiomer $\left(\mathrm{IC}_{50} \sim 100 \mathrm{nM}\right)$. Whereas the $\mathrm{IC}_{50}$ of $3 R, 5 S$-fluvastatin was between its typical unbound peak concentration in plasma and in the portal vein, that of $3 S, 5 R$-fluvastatin largely exceeded its clinically relevant unbound concentrations (Fig. 4). Likewise, the $\mathrm{IC}_{50}$ value of simvastatin acid $(19.7 \mathrm{nM})$ exceeded its $\mathrm{C}_{\max , \mathrm{u}}$ by 35 -fold. On the other hand, its concentrations in the portal vein may be higher. Furthermore, simvastatin acid is a substrate of the hepatic uptake transporter OATP1B1 in vivo (Pasanen et al., 2006), indicating that its intracellular hepatocyte concentrations are likely higher than those in the surrounding blood stream. For all other parent statins acids, the $\mathrm{IC}_{50}$ values obtained were close to or below their typical unbound peak concentrations in plasma and in the portal vein.

Taken together, we comprehensively investigated the in vitro metabolism and pharmacodynamics of statins. Together with drug transport and clinical data, our findings are applicable for use in systems pharmacology models to prospectively predict the pharmacokinetics and pharmacological effects of statins at different dosages.

\section{Acknowledgments}

The authors would like to thank Jouko Laitila for skillful technical assistance and help with the experiments.

\section{Authorship Contributions}

Participated in research design: Filppula, Hirvensalo, Parviainen, Niemi.

Conducted experiments: Filppula, Hirvensalo, Parviainen, Ivaska, Lönnberg, Deng, Viinamäki, Kurkela, Neuvonen.

Performed data analysis: Filppula, Hirvensalo, Parviainen, Ivaska.

Wrote or contributed to the writing of the manuscript: Filppula, Hirvensalo, Parviainen, Ivaska, Lönnberg, Deng, Viinamäki, Kurkela, Neuvonen, Niemi.

\section{References}

Billecke S, Draganov D, Counsell R, Stetson P, Watson C, Hsu C, and La Du BN (2000) Human serum paraoxonase (PON1) isozymes $\mathrm{Q}$ and $\mathrm{R}$ hydrolyze lactones and cyclic carbonate esters. Drug Metab Dispos 28:1335-1342.

Boberg M, Vrana M, Mehrotra A, Pearce RE, Gaedigk A, Bhatt DK, Leeder JS, and Prasad B (2017) Age-dependent absolute abundance of hepatic carboxylesterases (CES1 and CES2) by LC-MS/MS proteomics: application to PBPK modeling of oseltamivir in vivo pharmacokinetics in infants. Drug Metab Dispos 45:216-223.

Bradford RH, Shear CL, Chremos AN, Dujovne C, Downton M, Franklin FA, Gould AL, Hesney M, Higgins J, Hurley DP, et al. (1991) Expanded Clinical Evaluation of Lovastatin (EXCEL) study results. I. Efficacy in modifying plasma lipoproteins and adverse event profile in 8245 patients with moderate hypercholesterolemia. Arch Intern Med 151:43-49.

Corsini A, Maggi FM, and Catapano AL (1995) Pharmacology of competitive inhibitors of HMGCoA reductase. Pharmacol Res 31:9-27.

Dujovne CA, Chremos AN, Pool JL, Schnaper H, Bradford RH, Shear CL, Higgins J, Downton M, Franklin FA, Nash DT, et al. (1991) Expanded clinical evaluation of lovastatin (EXCEL) study results: IV. Additional perspectives on the tolerability of lovastatin. Am J Med 91 (1B):25S-30S. Fischer V, Johanson L, Heitz F, Tullman R, Graham E, Baldeck JP, and Robinson WT (1999) The 3-hydroxy-3-methylglutaryl coenzyme A reductase inhibitor fluvastatin: effect on human cytochrome P-450 and implications for metabolic drug interactions. Drug Metab Dispos 27:410-416.

Fujino H, Saito T, Tsunenari Y, Kojima J, and Sakaeda T (2004) Metabolic properties of the acid and lactone forms of HMG-CoA reductase inhibitors. Xenobiotica 34:961-971.

Fujino H, Yamada I, Shimada S, Yoneda M, and Kojima J (2003) Metabolic fate of pitavastatin, a new inhibitor of HMG-CoA reductase: human UDP-glucuronosyltransferase enzymes involved in lactonization. Xenobiotica 33:27-41.

Gertz M, Harrison A, Houston JB, and Galetin A (2010) Prediction of human intestinal first-pass metabolism of 25 CYP3A substrates from in vitro clearance and permeability data. Drug Metab Dispos 38:1147-1158.

Gertz M, Houston JB, and Galetin A (2011) Physiologically based pharmacokinetic modeling of intestinal first-pass metabolism of CYP3A substrates with high intestinal extraction. Drug Metab Dispos 39:1633-1642.

Giacomini KM, Balimane PV, Cho SK, Eadon M, Edeki T, Hillgren KM, Huang SM, Sugiyama Y, Weitz D, Wen Y, et al.; International Transporter Consortium (2013) International Transporter Consortium commentary on clinically important transporter polymorphisms. Clin Pharmacol Ther 94:23-26.

Graham DJ, Staffa JA, Shatin D, Andrade SE, Schech SD, La Grenade L, Gurwitz JH, Chan KA, Goodman MJ, and Platt R (2004) Incidence of hospitalized rhabdomyolysis in patients treated with lipid-lowering drugs. JAMA 292:2585-2590.

Hirvensalo P, Tornio A, Neuvonen M, Kiander W, Kidron H, Paile-Hyvärinen M, Tapaninen T, Backman JT, and Niemi M (2019) Enantiospecific Pharmacogenomics of Fluvastatin. Clin Pharmacol Ther 106:668-680.

Hoffmann M and Nowosielski M (2008) DFT study on hydroxy acid-lactone interconversion of statins: the case of atorvastatin. Org Biomol Chem 6:3527-3531.

Holdgate GA, Ward WH, and McTaggart F (2003) Molecular mechanism for inhibition of 3hydroxy-3-methylglutaryl CoA (HMG-CoA) reductase by rosuvastatin. Biochem Soc Trans 31:528-531.

Honda A, Mizokami Y, Matsuzaki Y, Ikegami T, Doy M, and Miyazaki H (2007) Highly sensitive assay of HMG-CoA reductase activity by LC-ESI-MS/MS. J Lipid Res 48:1212-1220.

Houston JB and Galetin A (2008) Methods for predicting in vivo pharmacokinetics using data from in vitro assays. Curr Drug Metab 9:940-951.

Itkonen MK, Tornio A, Neuvonen PJ, Niemi M, and Backman JT (2015) Clopidogrel does not significantly affect the pharmacokinetics of simvastatin: a crossover study in healthy volunteers. Clin Pharmacol Ther 97:S43-S43.

Ito K, Iwatsubo T, Kanamitsu S, Nakajima Y, and Sugiyama Y (1998) Quantitative prediction of in vivo drug clearance and drug interactions from in vitro data on metabolism, together with binding and transport. Annu Rev Pharmacol Toxicol 38:461-499. 
Jamei M (2016) Recent advances in development and application of physiologically-based pharmacokinetic (PBPK) models: a transition from academic curiosity to regulatory acceptance. Curr Pharmacol Rep 2:161-169.

Jemal M, Ouyang Z, Chen BC, and Teitz D (1999) Quantitation of the acid and lactone forms of atorvastatin and its biotransformation products in human serum by high-performance liquid chromatography with electrospray tandem mass spectrometry. Rapid Commun Mass Spectrom 13: $1003-1015$.

Johnson TN and Rostami-Hodjegan A (2011) Resurgence in the use of physiologically based pharmacokinetic models in pediatric clinical pharmacology: parallel shift in incorporating the knowledge of biological elements and increased applicability to drug development and clinica practice. Paediatr Anaesth 21:291-301.

Kantola T, Kivistö KT, and Neuvonen PJ (1998) Effect of itraconazole on the pharmacokinetics of atorvastatin. Clin Pharmacol Ther 64:58-65.

Kathawala FG (1991) HMG-CoA reductase inhibitors: an exciting development in the treatment of hyperlipoproteinemia. Med Res Rev 11:121-146.

Kenny JR, Mukadam S, Zhang C, Tay S, Collins C, Galetin A, and Khojasteh SC (2012) Drugdrug interaction potential of marketed oncology drugs: in vitro assessment of time-dependent cytochrome $\mathrm{P} 450$ inhibition, reactive metabolite formation and drug-drug interaction prediction. Pharm Res 29:1960-1976.

Keskitalo JE, Kurkinen KJ, Neuvonen M, Backman JT, Neuvonen PJ, and Niemi M (2009a) No significant effect of $\mathrm{ABCB} 1$ haplotypes on the pharmacokinetics of fluvastatin, pravastatin, lovastatin, and rosuvastatin. Br J Clin Pharmacol 68:207-213.

Keskitalo JE, Pasanen MK, Neuvonen PJ, and Niemi M (2009b) Different effects of the ABCG2 c.421C $>$ A SNP on the pharmacokinetics of fluvastatin, pravastatin and simvastatin. Pharmacogenomics 10:1617-1624.

Keskitalo JE, Zolk O, Fromm MF, Kurkinen KJ, Neuvonen PJ, and Niemi M (2009c) ABCG2 polymorphism markedly affects the pharmacokinetics of atorvastatin and rosuvastatin. Clin Pharmacol Ther 86:197-203.

Kitazawa E, Tamura N, Iwabuchi H, Uchiyama M, Muramatsu S, Takahagi H, and Tanaka M (1993) Biotransformation of pravastatin sodium (I). Mechanisms of enzymic transformation and epimerization of an allylic hydroxy group of pravastatin sodium. Biochem Biophys Res Commun 192:597-602.

Lehtisalo M, Keskitalo JE, Tornio A, Lapatto-Reiniluoto O, Deng F, Jaatinen T, Viinamaki J, Neuvonen M, Backman JT, and Niemi M (2020) Febuxostat, but not allopurinol, markedly raises the plasma concentrations of the breast cancer resistance protein substrate rosuvastatin. Clin Transl Sci 13:1236-1243.

Li Z, Zhang J, Zhang Y, and Zuo Z (2019) Role of esterase mediated hydrolysis of simvastatin in human and rat blood and its impact on pharmacokinetic profiles of simvastatin and its active metabolite in rat. J Pharm Biomed Anal 168:13-22.

Liederer BM and Borchardt RT (2006) Enzymes involved in the bioconversion of ester-based prodrugs. J Pharm Sci 95:1177-1195.

McCormick AD, McKillop D, Bulters CJ, Miles GS, Baba T, Touchi A, and Yamaguchi Y (2000) ZD4522: an HMG-CoA reductase inhibitor free of metabolically mediated drug interactions: metabolic studies in human in vitro systems (Abstract). J Clin Pharmacol 40:1055.

McTaggart F, Buckett L, Davidson R, Holdgate G, McCormick A, Schneck D, Smith G, and Warwick M (2001) Preclinical and clinical pharmacology of Rosuvastatin, a new 3-hydroxy-3-methylglutaryl coenzyme A reductase inhibitor. Am J Cardiol 87 (5A):28B-32B.

Neuvonen PJ, Niemi M, and Backman JT (2006) Drug interactions with lipid-lowering drugs: mechanisms and clinical relevance. Clin Pharmacol Ther 80:565-581.
Obach RS (1999) Prediction of human clearance of twenty-nine drugs from hepatic microsomal intrinsic clearance data: An examination of in vitro half-life approach and nonspecific binding to microsomes. Drug Metab Dispos 27:1350-1359.

Obach RS, Walsky RL, and Venkatakrishnan K (2007) Mechanism-based inactivation of human cytochrome p450 enzymes and the prediction of drug-drug interactions. Drug Metab Dispos 35:246-255.

Pasanen MK, Neuvonen M, Neuvonen PJ, and Niemi M (2006) SLCO1B1 polymorphism markedly affects the pharmacokinetics of simvastatin acid. Pharmacogenet Genomics 16:873-879.

Pazzucconi F, Dorigotti F, Gianfranceschi G, Campagnoli G, Sirtori M, Franceschini G, and Sirtori CR (1995) Therapy with HMG CoA reductase inhibitors: characteristics of the long-term permanence of hypocholesterolemic activity. Atherosclerosis 117:189-198.

Perchellet JP, Perchellet EM, Crow KR, Buszek KR, Brown N, Ellappan S, Gao G, Luo D, Minatoya M, and Lushington GH (2009) Novel synthetic inhibitors of 3-hydroxy-3-methylglutarylcoenzyme A (HMG-CoA) reductase activity that inhibit tumor cell proliferation and are structurally unrelated to existing statins. Int J Mol Med 24:633-643.

Prueksaritanont T, Subramanian R, Fang X, Ma B, Qiu Y, Lin JH, Pearson PG, and Baillie TA (2002) Glucuronidation of statins in animals and humans: a novel mechanism of statin lactonization. Drug Metab Dispos 30:505-512.

Rostami-Hodjegan A and Tucker G (2004) 'In silico' simulations to assess the 'in vivo' consequences of 'in vitro' metabolic drug-drug interactions. Drug Discov Today Technol 1:441-448.

Sager JE, Yu J, Ragueneau-Majlessi I, and Isoherranen N (2015) Physiologically based pharmacokinetic (PBPK) modeling and simulation approaches: a systematic review of published models, applications, and model verification. Drug Metab Dispos 43:1823-1837.

Thompson PD, Clarkson P, and Karas RH (2003) Statin-associated myopathy. JAMA 289: $1681-1690$.

Wang X, Zhu HJ, and Markowitz JS (2015) Carboxylesterase 1-mediated drug-drug interactions between clopidogrel and simvastatin. Biol Pharm Bull 38:292-297.

Varma MV, Bi YA, Kimoto E, and Lin J (2014) Quantitative prediction of transporter- and enzyme-mediated clinical drug-drug interactions of organic anion-transporting polypeptide 1B1 substrates using a mechanistic net-effect model. J Pharmacol Exp Ther 351:214-223.

Watanabe T, Kusuhara H, Maeda K, Shitara Y, and Sugiyama Y (2009) Physiologically based pharmacokinetic modeling to predict transporter-mediated clearance and distribution of pravastatin in humans. J Pharmacol Exp Ther 328:652-662.

Venkatakrishnan K and Rostami-Hodjegan A (2019) Come dance with me: transformative changes in the science and practice of drug-drug interactions. Clin Pharmacol Ther 105:1272-1278.

Vickers S, Duncan CA, Vyas KP, Kari PH, Arison B, Prakash SR, Ramjit HG, Pitzenberger SM, Stokker G, and Duggan DE (1990) In vitro and in vivo biotransformation of simvastatin, an inhibitor of HMG CoA reductase. Drug Metab Dispos 18:476-483.

Yamada I, Fujino H, Shimada S, and Kojima J (2003) Metabolic fate of pitavastatin, a new inhibitor of HMG-CoA reductase: similarities and difference in the metabolism of pitavastatin in monkeys and humans. Xenobiotica 33:789-803.

Yebyo HG, Aschmann HE, Kaufmann M, and Puhan MA (2019) Comparative effectiveness and safety of statins as a class and of specific statins for primary prevention of cardiovascular disease: A systematic review, meta-analysis, and network meta-analysis of randomized trials with 94,283 participants. Am Heart J 210:18-28.

Address correspondence to: Mikko Niemi, P.O. Box 20, 00014 University of Helsinki, Helsinki, Finland. E-mail: mikko.niemi@ helsinki.fi 